\title{
Sistema Hipermídia Adaptativo Educacional Baseado em Estilos de Aprendizagem
}

\author{
Rodrigo E. Carneiro ${ }^{1}$, Bruna A. Galle ${ }^{1}$, Fernando B. Lima Neto ${ }^{1}$, Denis S. Silveira ${ }^{2}$ \\ ${ }^{1}$ Escola Politécnica de Pernambuco - Universidade de Pernambuco (UPE) \\ Recife - PE - Brasil \\ ${ }^{2}$ Centro de Ciências Sociais Aplicada \\ Universidade Federal de Pernambuco (UFPE) - Recife, PE - Brasil \\ $\{$ rec, bcga, fbln\} @ecomp.poli.br, dsilveira@ufpe.br
}

\begin{abstract}
Adaptive Hypermedia Systems are systems that adapt according to the profile of each user. Nowadays, they represent an advancement over traditional tutoring systems. In this paper, it is proposed the use of Learning Styles (LS) during the process of content adaptation in order to increase the satisfaction of the learner during training. Its use was assessed qualitatively and it demonstrated wide acceptance.
\end{abstract}

Resumo. Sistemas Hipermídia Adaptativos são sistemas que se adaptam de acordo com o perfil de cada usuário. Seu uso com objetivo educacional vem crescendo e atualmente, eles representam um avanço em relação aos sistemas de tutoria tradicionais. Neste artigo é proposto a utilização de Estilos de Aprendizagem (EA) durante o processo de adaptação de conteúdo com o objetivo de aumentar a satisfação do aprendiz durante o treinamento e seu uso foi avaliado qualitativamente demonstrando grande aceitação.

\section{Introdução}

A facilidade de acesso aos computadores junto com a popularização da Internet impulsionaram o crescimento do uso da computação para fins educacionais em todo mundo [Brasil, 2012]. Atualmente, o Ensino a Distância (EAD) se tornou uma importante fonte de obtenção de conhecimento, fornecendo conteúdo sem a limitação de tempo ou espaços físicos.

Devido à grande variedade de perfis de usuários participantes desses cursos, torna-se cada vez mais difícil manter a qualidade e satisfação dos alunos com o modelo tradicional de ensino, já que o mesmo conteúdo é exibido para todos os participantes independentemente de suas idiossincrasias [Schnitman, 2010].

Os sistemas hipermídia adaptativos combinam ideias dos sistemas hipermídia e dos sistemas tutores inteligentes [Brusilovsky \& Nejdl, 2003]. Ou seja, sistemas que sabem o que ensinar (conteúdo), para quem ensinar (modelagem do aluno) e como ensinar (estratégias pedagógicas ou de ensino) [Silva, 2000].

Neste trabalho se utiliza Estilos de Aprendizagem (EA), extraídos com o questionário Felder-Silverman [Felder \& Silverman, 1988], para a adaptação do conteúdo as características do aprendiz utilizando um sistema hipermídia adaptativo. 


\section{Trabalhos Relacionados}

Várias pesquisas já foram feitas considerando as diferenças individuais dos aprendizes e o impacto disso no seu aprendizado.

[Bajraktarevic, Hall, \& Fullick, 2003] realizaram um estudo onde provaram que alunos frequentadores de cursos online que levam em consideração seu estilo de aprendizagem favorito (sequencial ou global) alcançaram resultados significativamente melhores do que aqueles que fizeram um curso onde tal preferência não era importante no processo de aprendizagem.

TANGOW [Paredes \& Rodriguez, 2004], e AHA! [Stash, Cristea, \& De Bra, 2006] são alguns exemplos de sistemas que incorporam EA e oferecem cursos que se encaixam com o perfil dos alunos.

\section{Fundamentação Teórica}

\subsection{Sistema hipermídia adaptativo}

Sistemas Hipermídia Adaptativos (SHA) [Brusilovsky \& Nejdl, 2003] são sistemas capazes de se adaptarem aos usuários. Quando usados com foco educacional, combinam ideias de sistemas hipermídia e sistemas tutores. Comumente, os SHAs são constituídos pelos seguintes componentes [Brusilovsky \& Nejdl, 2003]:

- Modelo do Usuário (MU): Componente responsável por representar o conhecimento do usuário do sistema. Essas informações são necessárias para a adaptação do conteúdo;

- Modelo de Adaptação (MA): Responsável pela escolha das táticas e estratégias pedagógicas que serão aplicadas para o treinamento conforme os dados obtidos a partir do estado atual do MU;

- Modelo Domínio (MD): É o módulo especialista do sistema, responsável por gerenciar todo material instrucional que será apresentado ao aprendiz;

- Modelo Interface (MI): É o meio de interação entre o SHA e os usuários.

A partir da interação do usuário com o MI, é possível criar sua representação de conhecimento no MU, o que possibilita que os conteúdos do MD possam ser adaptados pelo MA. Portanto, a MU é fundamental para uma boa adaptação do sistema e por esse motivo é crescente alvo de estudo.

\subsection{Estilos Cognitivos de Aprendizagem}

Estudantes aprendem de muitas maneiras, como por exemplo, vendo e ouvindo; refletindo e atuando, entre outros. Estas maneiras ou estilos de aprendizagem podem ser representados por diversos modelos desenvolvidos por vários autores como Kolb [Kolb, 1974], Felder-Silverman [Felder \& Silverman, 1988] etc.

Cada autor utiliza diferentes dimensões para classificar as características dos aprendizes, por exemplo:

- Felder-Silverman (1988): abrange quatro dimensões da aprendizagem: Percepção (Sensorial/Intuitiva), Entrada (Visual/Verbal), Processamento (Ativo/Reflexivo) e Entendimento (Sequencial/Global); 
- Kolb (1973): é definido de acordo com duas escalas: experiência concreta ou conceitualização abstrata e experimentação ativa ou observação reflexiva.

A modelagem é feita a partir de um questionário de preferências e seu resultado representa a forma mais agradável e eficiente de aprendizado para um dado usuário.

Em [Valaski, Malucelli, \& Reinehr, 2011], identifica-se que o modelo mais referenciado no período de 2005 a 2011 seria o modelo Felder-Silverman. E por este motivo foi o escolhido para aquisição do EA no sistema adaptativo proposto.

\section{Metodologia}

Para a construção do EA do aprendiz foi utilizado o questionário Felder-Silverman que contém 44 questões e classifica-o em quatro dimensões cognitivas (Percepção, Entrada, Processamento e Entendimento) [Felder \& Silverman, 1988].

Cada dimensão é avaliada por 11 questões podendo ser classificada de duas formas diferentes. Por exemplo, a dimensão de Entrada pode ser classificada como Verbal e Visual que por sua vez tem sua intensidade mensurada pela quantidade de respostas de cada tipo. Resultados de 1 ou 3 em determinado domínio indica fraca preferência e equivale dizer que o aprendiz está balanceado entre as duas opções; um resultado de 5 ou 7 indica preferência moderada; e um resultado de 9 ou 11 indica forte preferência ao tipo de aprendizagem.

O aprendiz após a resolução do questionário tem seu EA armazenado e este será utilizado na personalização do conteúdo apresentado pelo sistema adaptativo.

Para cada dimensão é calculado quantos por cento o aprendiz é representado pelo estilo de aprendizagem predominante. Por exemplo, um aprendiz que possuir na dimensão de Entrada a característica visual predominante, por ter respondido 7 das 11 questões referentes a este estilo, terá $64 \%$ visual e $36 \%$ para o estilo verbal.

O mecanismo adaptativo proposto utiliza uma roleta de probabilidade para decidir qual tipo de conteúdo será exibido ao aprendiz baseando-se em seu EA. Se o número sorteado na roleta for inferior ao valor da porcentagem referente à preferência em uma dada dimensão, será apresentado um conteúdo com características do estilo de aprendizagem dominante, enquanto quando sorteado um valor superior ao valor da do EA, é selecionado um conteúdo do estilo de aprendizagem não dominante.

\section{Aplicação Experimental}

Foi proposto um estudo empírico do tipo experimento voluntário, realizado online onde os participantes foram convidados por email ou mensagens em redes sociais, que tem como objetivo analisar qualitativamente o uso da adaptação por EA em um SHA.

Para o experimento foram utilizados conteúdos, MD, sobre o editor de texto Microsoft Word construídos de 2 formas diferentes. A primeira, utilizando imagens e textos (Figura 1a) enquanto a segunda fazia uso de vídeos sobre o mesmo tema (Figura 1b). Para o MU, foi aplicado o questionário Felder-Silverman para modelagem do EA.

Baseando-se na dimensão de entrada do EA de cada usuário, o sistema utilizou, como MA, a roleta de probabilidade para decidir qual tipo de tela seria exibida ao aprendiz. Para o estilo de aprendizagem verbal foram apresentados imagens e textos enquanto para o estilo visual foram apresentados os conteúdos em vídeo. 
Após o treinamento no sistema adaptativo, o aprendiz respondeu um questionário qualitativo com intuito de verificar sua opinião e sugestões de melhorias.

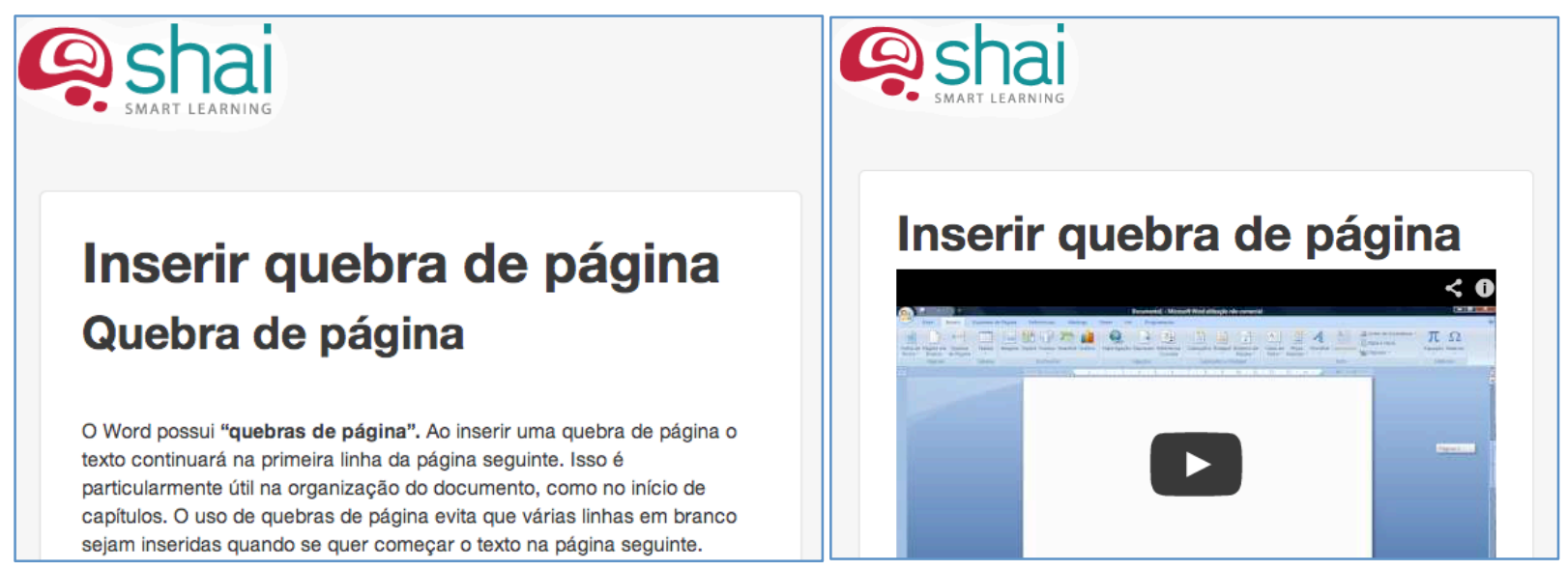

Figura 1. Exemplo de telas utilizadas em perfis com preferência verbal e visual respectivamente.

\section{Resultados}

Durante o experimento, foram armazenados de cada participante seu cadastro pessoal, seu EA e suas respostas do questionário qualitativo. $O$ experimento contou com a participação de 71 aprendizes entre 18 e 50 anos, onde $63 \%$ deles pertenciam ao sexo masculino e $37 \%$ feminino.

O estilo de aprendizagem mais frequente entre os participantes na dimensão de Processamento foi o Ativo com $76 \%$ dos aprendizes encaixando-se nesta característica; na dimensão Percepção, o estilo Sensorial obteve $71 \%$ do total de participantes; na dimensão de Entrada, o estilo Visual foi o mais ocorrido com $78 \%$, e por fim, na dimensão Organização, 53\% ficaram com o estilo Sequencial.

A grande maioria dos usuários consideraram o sistema hipermídia adaptativo fácil de usar e concordaram que a apresentação adaptativa facilitou a aprendizagem. Sobre o questionário Felder-Silverman, a grande maioria considerou muito longo, porém direto e de fácil entendimento. $70 \%$ dos participantes consideraram excelente a adaptação por estilo de aprendizagem e $85 \%$ afirmaram que gostariam de utilizar esta ferramenta para aprender outros tipos de conteúdo.

\section{Conclusão e Trabalhos Futuros}

Este trabalho teve como objetivo utilizar Estilos de Aprendizagem, extraídos utilizandose o questionário Felder-Siverlman, a fim de adaptar o conteúdo às características do aprendiz utilizando um sistema hipermídia adaptativo. Os resultados obtidos mostramse promissores, considerando que a grande maioria dos participantes gostaram da adaptação do conteúdo ao seu EA e gostariam de fazer outros treinamentos adaptativos.

Em trabalhos futuros, pretende-se analisar a possibilidade de redução do tamanho do questionário de preferências, além de criar outras dimensões cognitivas 
para representar o aprendiz e também novos mecanismos adaptativos, por exemplo, a utilização o desempenho do aprendiz para controle de progresso do treinamento. E por fim, integrar a um processo de avaliação de aprendizado baseada em IA.

\section{Referências}

Bajraktarevic, N., Hall, W., \& Fullick, P. (2003). Incorporating learning styles in hypermedia environment: Empirical evaluation. In Proceedings of the Workshop on Adaptive Hypermedia and Adaptive Web-Based Systems (pp. 41-52). Nottingham, UK.

Brasil, C. G. da I. no. (2012). Pesquisa sobre o uso das tecnologias de informação e comunicação no Brasil : TIC Educação 2011. (A. F. Barbosa, Ed.) (p. 400). São Paulo. Retrieved from http://op.ceptro.br/cgi-bin/cetic/tic-educacao-2011.pdf

Brusilovsky, P., \& Nejdl, W. (2003). Adaptive hypermedia and adaptive web. In M. P. Singh (Ed.), Practical Handbook of Internet Computing. CRC Press. Retrieved from http://www.kbs.uni-hannover.de/Arbeiten/Publikationen/2003/brusilovskynejdl.pdf

Felder, R. M., \& Silverman, L. K. (1988). Learning and Teaching Styles in Engeneering Education. Journal of Engineering Education, 78(June), 674-681. Retrieved from http://www.researchgate.net/publication/236261199_Comparative_Education_Tea cher_Training_Education_Policy_School_Leadership_and_Social_Inclusion/file/6 0b7d5176c814cd615.pdf\#page $=407$

Kolb, D. (1974). On management and the learning process. Cambridge, Mass.: M.I.T. Retrieved from http://psycnet.apa.org/psycinfo/1974-33290-011

Paredes, P., \& Rodriguez, P. (2004). A mixed approach to modelling learning styles in adaptive educational hypermedia. In International Conference on Webbased Education (Vol. 1, pp. 210-215).

Schnitman, I. M. (2010). O perfil do aluno virtual e as teorias de estilos de aprendizagem. In $3^{\circ}$ Simpósio Hipertexto e Tecnologias na Educação: redes sociais e aprendizagem (Vol. 1, pp. 1-10).

Silva, A. do S. da. (2000). TUTA - Um Tutor Baseado em Agentes no Contexto do Ensino a Distância. UFPB.

Stash, N., Cristea, A., \& De Bra, P. (2006). Adaptation to learning styles in e-learning: Approach evaluation. In World Conference on E-Learning in Corporate, Government, Healthcare, and Higher Education (pp. 284-291).

Valaski, J., Malucelli, A., \& Reinehr, S. (2011). Revisão dos Modelos de Estilos de Aprendizagem Aplicados à Adaptação e Personalização dos Materiais de Aprendizagem. Simpósio Brasileiro de Informática na Educação, 2-5. Retrieved from http://www.br-ie.org/pub/index.php/sbie/article/view/1843 